\title{
An update on neurocognitive impairment in schizophrenia and depression
}

\author{
Kenneth Hugdahl1,2* and Vince D. Calhoun ${ }^{3,4 * *}$ \\ 1 Department of Biological and Medical Psychology, University of Bergen, Bergen, Norway \\ 2 Division of Psychiatry, Haukeland University Hospital, Bergen, Norway \\ 3 The Mind Research Network, Albuquerque, NM, USA \\ 4 Department of ECE, University of New Mexico, Albuquerque, NM, USA \\ ${ }^{*}$ Correspondence: *hugdahl@psybp.uib.no; **vcalhoun@unm.edu
}

Despite the considerable research that has been devoted to schizophrenia and depression over the last years, the understanding of the biological bases of these disabling disorders is still fragmentary. Adoption-, twin- and family studies, provide clear evidence of strong genetic components to these mental disorders, although multiple loci are likely to be involved. The effect of pharmacological intervention has hinted at specific neurotransmitter systems of etiological importance, but the lack of receptor specificity of therapeutic drugs have thwarted any simple linkage of behavioural and molecular phenotypes. More recently brain imaging investigations, like PET and functional magnetic resonance imaging (fMRI), have identified candidate structural and functional abnormalities, including work in identifying changes in networks of brain regions, but these findings are still preliminary and need to be integrated with other accumulating knowledge.

Of particular interest in the last decade that is adding to the complexity of the picture, is the realization that basic cognitive functions are seriously distorted, and that removal of core symptoms such as paranoia and hallucinations does not necessarily ameliorate the cognitive deficits. It has also been shown that cognitive deficits can occur before clinical symptoms in schizophrenia, in a prodromal phase of the disorder, pointing to a possible causal relationship between cognitive deficits and outbreak of the disorder. Similarly, it has been reported that removing clinical symptoms in depression does not alleviate cognitive symptoms, like impaired concentration and focus of attention. However, it is still not possible to diagnose a patient from cognitive deficits alone, or from a combination of clinical symptoms and cognitive deficits, and structural and functional neuroimaging data, although promising, have also not found the way to routine clinical practice.

The aim of the current special topic is to provide an update on research on cognitive and brain imaging research for the understanding of cognitive deficits in these disorders, with a focus on recent developments in structural and functional neuroimaging, including recent advances in tracking of white matter fibres, and electroencephalography (EEG) and novel computational measures.

Vince Calhoun et al. show how fMRI can be used not only to identifying regions where hemodynamic response amplitudes can differentiate between patient and control groups, but also how such amplitude based comparisons can estimate temporal correlations and compute maps of functional connectivity between regions. In their article, Calhoun et al. review work related to how these brain networks are pervasive also in a relaxed resting state, included the default mode network and how such analysis can provide novel approaches to yielding biomarkers for schizophrenia and other mental disorders, which can be described both in terms of disrupted local processing as well as altered global connectivity between large scale cortical networks. The paper also introduces new statistical approaches to functional connectivity, emphasizing the use of independent component analysis.

Bjørn Rishovd Rund reviews recent research and discusses whether schizophrenia is a neurodegenerative disorder, with progressive neuronal degeneration as the illness progresses. Acknowledging that this issue has been discussed ever since the identification of schizophrenia as a disorder of its own, Rund focuses on what can be learned from recent cognitive and brain imaging data. He takes the position that although there are reports of cognitive decline the longer the patient has been ill, and corresponding grey matter changes, there is no convincing evidence of a progressive neurodegenerative process after onset of illness. He further posits that MRI and cognitive changes can be explained by reference to compensatory mechanisms and cognitive reserve theory after the onset of schizophrenia, at the same time as cognitive functioning does not get increasingly impaired in the course of the disorder.

Dara Manoach and Robert Stickgold raises the often neglected and poorly understood issue of sleep disorders in schizophrenia and ask the important question if abnormal sleep impair memory consolidation in schizophrenia? Starting with the observation that sleep abnormality often impairs cognitive functioning in healthy individuals it is imperative to delineate whether a similar relationship exists in mental disorders where cognition is affected. Manoach and Stickgold suggests that the evolution of memory consolidation over time is facilitated by sleep, and they then review existing data that abnormal sleep in schizophrenia disrupts attention and impairs sleep-dependent memory consolidation and task automation. The authors conclude that these sleep-dependent impairments may contribute substantially to the generalized cognitive deficits in schizophrenia and that focus on the amelioration of sleep disturbances may have important consequences for clinical improvement.

Åsa Hammar and Guro Årdal review their work on cognitive function in patients with major depression, and the question if repeated relapses of depressed episodes increasingly impair cognition and if cognition is similarly restored after symptom treatment and restoration of normal functioning. Hammar and Årdal review recent research that has focused on cognitive functioning in the severe phase of depression, where it is widely accepted that the disease is characterized by cognitive impairment in the acute state. 
They then go on to review their own work that has used an experimental approach focusing on attention shift and attentive search processes. They show that cognitive impairment remains during depressed episodes despite clinical improvement. The authors also show that impaired ability to perform the attention tasks employed in their studies is related to frequency of depressed episodes and with the duration of episodes.

Else-Marie Løberg and Kenneth Hugdahl review available studies on a relationship between cannabis use and cognitive functioning in schizophrenia. It is an established fact that cannabis use is quite frequent in schizophrenia. It has also been suggested that cannabis actually can be a contributing causal factor in schizophrenia, which may interact with the typical neurocognitive vulnerability seen in this patient group. Løberg and Hugdahl reviews the literature and of the 23 studies that were found, a clear majority reported either improved cognitive functioning or no change, with only one study reporting a decline. These paradoxical findings may be explained by several alternative hypotheses. One hypothesis is that the cannabis group overall has a better functioning with superior social skills, enabling drug acquisition. Another hypothesis is that cannabis use could have a positive influence on brain functioning, but taking the negative effects of cannabis on brain and psychosis into account, this is not probable. A cannabis-related schizophrenia group study show poorer prognosis and studies on premorbid functioning are inconclusive. The authors speculate that cannabis may, through a breakdown of brain function, imitate the typical neurocognitive vulnerability seen in schizophrenia.

Jazmin Camchong et al. present two studies from their laboratory where they have used diffusion tensor MR imaging (DTI) to obtain brain anatomical connectivity information in patients with schizophrenia and healthy controls by examining the directional organization of white matter microstructure. The authors use DTI to address the important question of whether brain connectivity and its abnormalities may be heritable traits associated with schizophrenia. The first study investigated if healthy monozygotic (MZ) twin pairs and random pairings among twins would show different correlations with regard to DTI measures of neuronal connectivity. The results showed that there was a stronger correlation between $\mathrm{MZ}$ twin pairs than between randomly generated pairs in several regions in the brain critically involved in cognitive function. In a second study the same approach was applied to first-degree relatives of schizophrenia patients and a healthy control group. The results showed that relatives of schizophrenia patients had reduced connectivity in brain regions involved in controlling higher cognitive functions. The authors suggest that a DTI connectivity approach may be a valuable approach to unravelling new endophenotypes in schizophrenia.

Kevin Spencer provides data from a neuro-computational approach to elucidate the question of functional consequences of cortical circuit abnormalities on gamma oscillations in schizophrenia. In particular Spencer uses a computational model of cortical circuitry to examine the effects that neural circuit abnormalities might have on EEG gamma frequency generation and network excitability. The results showed that reducing synaptic connectivity (e.g. NMDA receptor input) had a negative effect on gamma frequency power and phase synchrony. Reducing neuronal spiking activity output impaired gamma generation to a lesser degree than reducing synaptic connectivity, thereby increasing network excitability. It is suggested that a neuro-computation approach, combining non-invasive neurophysiological and structural measures, might be able to distinguish between different neural circuit abnormalities in schizophrenia patients. Computational modelling may help to bridge the gaps between post-mortem studies, animal models, and experimental data in humans, and facilitate the development of new therapies for schizophrenia and neuropsychiatric disorders in general.

Godfrey Pearlson and Vince Calhoun ask why endophenotypes are important in schizophrenia, and suggest a distinction between definitions of endophenotypes versus biomarkers and the implications of this for the understanding of neurocognitive deficits. A starting point for their discussion is that because the pathophysiology of schizophrenia is obscure, there is no laboratory test or biological marker deriving from the core etiopathology that can be reliably used in diagnosis and treatment outcome evaluation. Pearlson and Calhoun then review existing data on the relationship between abnormal brain functioning and cognitive functioning, focusing on activation in the dorsolateral prefrontal cortex and adjacent regions and working memory, an often implied endophenotype. The authors identifies several problems with current approaches to the study of endophenotypes, such as patients not understanding the tasks, or task fatigue, and suggest alternative approaches such as simpler experimental tasks that might avoid difficult to interprate floor effects. They also advocate the use of resting state and default mode network paradigms, applying independent component analysis techniques to reveal functional networks rather than isolated brain regions when searching for endophenotypes and biological markers of schizophrenia.

Emma Thomas and Rebecca Elliott review work related to recent research on the neural basis of cognition in depression, focusing on the distinction between emotional and non-emotional processing. Cognitive deficits are among the core deficits in depression and also listed in recent diagnostic manuals as core symptoms, in particular reduced concentration and poor memory. Another equally important class of cognitive processes is the investigation of mechanisms of emotional disturbance, such as negative bias of thoughts and excessive response to failure. The article reviews the major emotional and non-emotional cognitive processes in depression, together with findings from neuroimaging studies. They conclude on the basis of their review of the data that models of cognitive dysfunction in depression is underscored by disrupted cortico-limbic circuitry, and discuss how connectivity analysis MRI techniques can be used to test such models explicitly, revealing important clinical implications of cognitive imaging in depression. Understanding of such relationships may have important consequences for diagnosis and treatment of depression and depressive episodes.

Matcheri Keshavan and colleagues review previous work on premorbid cognitive deficits and their relationship to brain structure in young relatives of schizophrenia patients. They note cognitive deficits in the adolescent population which also manifest in firstdegree relatives. The primary deficits include psychomotor speed, memory, attention, reasoning, and social-cognition in addition to generalized measures such as intelligence. Of note, the age of adolescence may be a useful "window" for observing premorbid 
impairments in neurocognition. They also examine the relationship between brain structure and cognitive measures. There is much evidence of neurocognitive deficits, but more study is needed to improve our understanding of their structural underpinnings. In addition the potential to use these measures as biomarkers and possibly endophenotypes should be explored further.

Daniel Mathalon and colleagues present a study of electrophysiological abnormalities in schizophrenia and schizoaffective disorder (SA). In particular, P300 differences including amplitude and latency have been widely studied in schizophrenia but have not been well studies in SA. Interestingly they found that although P300 amplitude was preserved in the SA group the latency and reaction times were affected similarly in schizophrenia and schizoaffective disorder. These changes could not be accounted for by other measured demographic variables and suggests that the underlying pathophysiology of the two groups is different.

Sophia Frangou provides a selective review of cognitive function in early onset schizophrenia. Changes in IQ, attention, executive function and memory have been observed and in this group, which provides a unique way to study the trajectory of the disease in a more severe variant of adult onset schizophrenia. Frangou identified a consistent pattern of abnormalities in the various cognitive domains. In addition to finding evidence that the cognitive profile of the early onset group was similar to that of the less severe adult onset group she highlighted age related improvements which existed for healthy adolescents but not for patients.

Leighton Hinkley and colleagues present a magnetoencephalography (MEG) study of the neural connectivity in schizophrenia patients. They present several approaches for maximizing the sensitivity of the MEG technique including adaptive spatial filtering, different functional connectivity metrics, and study the relationship to various cognitive and clinical outcomes. The use of MEG can be used to augment existing work by adaptive existing paradigms used in EEG and fMRI to MEG. Finally they discuss experimental paradigms which demonstrate important utility in relating impaired connectivity to characteristic findings in schizophrenia.

In summary, the current collection of articles represents the wide range of topics related to cognitive impairment in schizophrenia and depression. In this respect the special topic issue provides compelling evidence that cognitive impairment is a primary aspect of both schizophrenia and depression, and not secondary to other symptoms and disease processes. The current selection of articles also show how recent developments in structural and functional neuroimaging, including both hemodynamic and electrophysiology, may further advance our understanding of how cognition is impaired in these disorders. The more complete picture of these disorders provided by cognitive measures, neuroimaging, and also genetics will likely be important tools in future diagnostics and treatment evaluation.

Received: 19 August 2009; accepted: 04 January 2010; published online: 24 February 2010. Citation: Front. Hum. Neurosci. (2010) 4:4. doi: 10.3389/neuro.09.004.2010

Copyright $\odot 2010$ Hugdahl and Calhoun. This is an open-access publication subject to an exclusive license agreement between the authors and the Frontiers Research Foundation, which permits unrestricted use, distribution, and reproduction in any medium, provided the original authors and source are credited. 